\title{
Staphylococcal toxins and sudden infant death syndrome
}

\author{
J E Malam, G F Carrick, D R Telford, J A Morris
}

\begin{abstract}
Aims: To investigate the hypothesis that commonly occurring bacterial toxins cause sudden infant death syndrome (SIDS) by (1), determining in which tissues bacterial toxins are concentrated after intravenous injection in rats; and (2), seeing if the same tissues contain detectable toxins in cases of SIDS.

Methods: The tissue distribution of intravenously injected staphylococcal enterotoxin A (SEA), enterotoxin B (SEB), enterotoxin C (SEC), enterotoxin D (SED), toxic shock syndrome toxin (TSST-1), and $a$-haemolysin was studied in rats using immunohistology and polyacrylamide gel electrophoresis with immunoblotting. Immunostaining was also carried out on formalin fixed kidneys from cases of SIDS and a comparison series of necropsy cases using anti-SEA, anti-SEB, anti-SEC ${ }_{2}$ and anti-SED.

Results: Immunohistology showed that SEB, SEC, SED and TSST-1 were all concentrated in the proximal convoluted tubular cells of the kidney. The presence of these toxins was confirmed in kidney homogenates using electrophoresis and immunoblotting. There was positive granular staining in the proximal convoluted tubular cells of the kidney in $36 \%$ of SIDS cases and $12 \%$ of the comparison series with anti SEC $_{2}\left(\chi^{2}=6\right.$; p $\left.<0.025\right)$.

Conclusion: SEC, or a bacterial toxin with epitopes in common, could have a pathogenic role in SIDS.
\end{abstract}

The idea that commonly occurring bacterial toxins have a pathogenic role in some cases of sudden infant death syndrome (SIDS) is gaining support. It is consistent with the epidemiological features of SIDS, including the winter excess of cases, the characteristic age distribution, and the association with a prone sleeping position. ${ }^{1-3}$ There is also experimental support from a number of studies of the nasopharyngeal ${ }^{4}$ and gut flora, ${ }^{5-7}$ and from animal models of bacterially induced sudden death. $^{8-10}$

In a recent immunohistological study we showed that staphylococcal toxic shock syndrome toxin (TSST-1) is concentrated in the proximal convoluted tubular cells of the kidney in rats following intravenous injection. ${ }^{11}$ The same antiserum gave positive staining in the proximal convoluted tubular cells of human kidney in $18 \%$ of SIDS cases. We now describe the results of work designed to extend these observations.

\section{Methods}

TOXIN PREPARATIONS

Samples of Staphylococcus aureus from the nasopharynges of babies attending a well baby clinic were phage typed and tested for toxin production at the Food Hygiene Laboratory, Central Public Health Laboratory, Colindale. The methods of obtaining the samples and the results obtained have been published elsewhere. ${ }^{4}$ Bacteria which produced one or more of the enterotoxins A, B, C, D and TSST-1 were used for subsequent study. Alpha-haemolysin was obtained from Wood 46 strain purchased from the Central Public Health Laboratory.

Bead cultures stored at $-70^{\circ} \mathrm{C}$ were grown on blood agar overnight at $37^{\circ} \mathrm{C}$, then transferred to bijou bottles containing $5 \mathrm{ml}$ brainheart infusion (BHI) medium, and again left overnight at $37^{\circ} \mathrm{C}$. Two drops of the culture were spread on dialysis membrane overlying BHI- $1.5 \%$ agar plates and incubated for three to four days at $37^{\circ} \mathrm{C}$ in $5 \% \mathrm{CO}_{2}$. Phosphate buffered saline (PBS) (2 ml) was used to wash the growth from the membrane and the suspension was centrifuged at $4000 \times g$ for 15 minutes. The supernatant was sterilised by passing through a $0.2 \mu \mathrm{m}$ Minisart disposable filter and stored at $-20^{\circ} \mathrm{C}$.

ELECTROPHORESIS AND IMMUNOBLOTTING

Toxin preparations were examined by sodium dodecyl sulphate polyacrylamide gel electrophoresis (SDS-PAGE), using a $1.5 \mathrm{~mm}$ slab gel containing $12 \%(\mathrm{~W} / \mathrm{V})$ acrylamide for the separating gel and a $4 \%$ acrylamide for the stacking gel. The buffer system was a slightly modified version of Laemmli. ${ }^{12}$ The sample buffer contained $2 \%$ SDS, $10 \%$ sucrose, 0.02 M TRIS-HCl (pH 8.0), 0.002 M EDTA and $2 \mathrm{mg}$ Pyronin $\mathrm{Y}$ as marker dye. (Mercapto-ethanol was added to the sample buffer for most toxins but was found to break down SEB into small fractions.) Equal quantities of sample and sample buffer were used at $25 \mu \mathrm{m} /$ well.Electrophoresis was carried out in "The Sturdier" vertical slab unit, SE 400 (Hoefer Scientific Instruments).

Immediately after electrophoresis the separating gels were soaked in transfer buffer (0.25 M TRIS, $0.192 \mathrm{M}$ glycine in $20 \%$ methanol) for 15 minutes before blotting. The separated proteins were transferred to nitrocellulose membrane for one hour at $100 \mathrm{~mA}$, 
using the TE 70 Semiphor semi-dry transfer unit (Hoefer Scientific Instruments). After transfer the nitrocellulose membrane was immersed in PBS-5\% normal swine serum (NSS) overnight at $4^{\circ} \mathrm{C}$. The following day the membrane was incubated in turn with the relevant polyclonal rabbit antitoxin at 1 in 1000 in PBS $-5 \%$ NSS for one hour at $37^{\circ} \mathrm{C}$, swine anti-rabbit antibody at 1 in 500 PBS-5\% NSS for one hour at $37^{\circ} \mathrm{C}$, and horseradish peroxidase complexed with rabbit anti-peroxidase at 1 in 1000 in PBS-5\% NSS for one hour at $37^{\circ} \mathrm{C}$. Between each incubation the membrane was washed three times for 10 minutes in PBS. The final washes were 10 minutes each in PBS, distilled water, and $0.05 \mathrm{M}$ acetate buffer ( $\mathrm{pH} \mathrm{5.0)}$, followed by visualisation of the antigen and complexed antibody with $0.06 \mathrm{~g}$ 3-amino9-ethyl carbazole (AEC) in $15 \mathrm{ml}$ dimethyl formamide diluted to $300 \mathrm{ml}$ with acetate buffer plus 5 drops of $30 \%$ hydrogen peroxide. The primary antisera used were directed against staphylococcal enterotoxins $\mathrm{A}, \mathrm{B}, \mathrm{C}_{1}, \mathrm{C}_{2}, \mathrm{C}_{3}, \mathrm{D}$ a-haemolysin and TSST-1 (Toxin Technology, Florida, USA). Comparison gels were stained with Coomassie blue.

ANIMAL TISSUE PREPARATION

Female Wistar rats weighing $240-330 \mathrm{~g}$ were anaesthetised by intraperitoneal injection of Nembutal $(0.75 \mathrm{ml} / 100 \mathrm{~g}$ body weight of a solution of $10 \%$ Nembutal in $10 \%$ ethyl alcohol). The toxin preparation from a single organism, which varied in volume from $1.0-1.5 \mathrm{ml}$ was given by slow intravenous injection into the tail vein of one test animal. An equal volume of sterile saline was injected into control animals. A total of 42 toxin preparations were tested with at least five repetitions of each toxin. (Some preparations contained more than one toxin). The rats were observed either until death or until the beginning of recovery from the anaesthetic. The rats were then sacrificed by cervical dislocation. Urine samples were collected if possible. Dissection was carried out immediately, and samples of kidney, heart, lung, liver and spleen were obtained. From some animals, a portion of gut, skin and thymus was also removed. A portion of each sample was stored frozen at $-20^{\circ} \mathrm{C}$ and the rest fixed in $10 \%$ formalin.

\section{IMMUNOSTAINING OF TISSUES}

Immunostaining was performed on formalin fixed, paraffin wax embedded blocks. A standard avidin-biotin peroxidase technique was used. The appropriate polyclonal rabbit antisera at a dilution of 1 in 300 for anti-B and anti- $C_{2}, 1$ in 150 for anti-A and anti-D and 1 in 240 for $a$-haemolysin was applied for three hours, followed by biotinylated swine antirabbit antibody at a dilution of 1 in 400 for 30 minutes, then avidin-biotin complex for 30 minutes. The antigen and complexed antibody were visualised with AEC, as previously described. For each test animal and its paired control the primary antisera were appropriate to the toxin given. In addition, a selection of positively stained kidneys were tested with all antisera to check for cross-reaction. In all cases the sections from both test and control animals were stained in duplicate with one acting as a negative control in which specific antiserum was replaced by normal rabbit serum (immunoglobulin fraction) at a similar protein dilution.

Selected tissues from the test and control rats were examined by electrophoresis and immunoblotting as described above. Approxi-

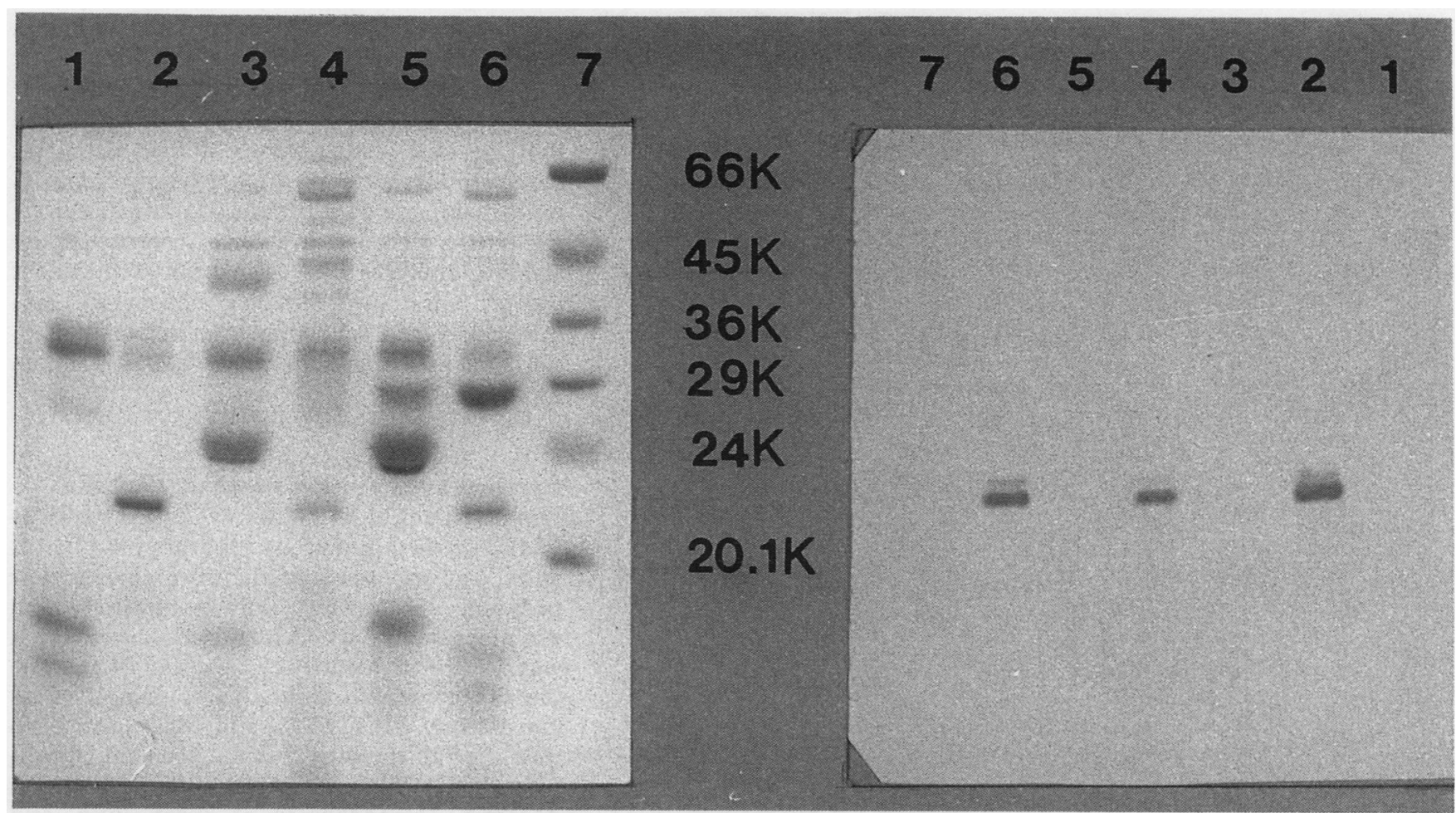

Figure 1 SDS-PAGE of staphylococcal products (lanes 1 to 6) on the left with a mirror image immunoblot stained with an antiserum to TSST-1 on the right. Lane 7 contains molecular weight markers. The staphylococci in lanes 2, 4 and 6 produce TSST-1 of molecular weight 22000. 
mately $1 \mathrm{~g}$ of tissue was frozen, slowly thawed and ground. PBS $(2 \mathrm{ml})$ were added and the freezing, thawing, and grinding repeated up to three times. The resulting suspension was centrifuged at $4000 \times g$ for 30 minutes and the supernatant filtered through a $0.2 \mu \mathrm{m}$ filter and stored at $-20^{\circ} \mathrm{C}$.

\section{Results}

ELECTROPHORESIS OF BACTERIAL PRODUCTS

The mixtures of bacterial toxins produced by growth of staphylococci on cellophane over agar were examined by SDS-PAGE followed by immunoblotting (fig 1). There was clear separation of the polypeptides secreted by the staphylococci. The TSST-1 band could easily be identified by its position which corresponds to a molecular weight of 22000 daltons. The toxins, SEA, SEB, SEC, and SED have molecular weights in the range $26000-30000$, as do many other products of the bacteria, therefore they could not be distinguished by their position alone. During the course of the work it was found that incubating the bacteria in a $\mathrm{CO}_{2}$ enriched atmosphere increased toxin production, as assessed by staining intensity of the bands on electrophoresis.

Polypeptide bands on electrophoresis

\begin{tabular}{llllllll}
\hline Antisera & 35000 & SEA & SEB & SEC & SED & 24000 & TSST-1 \\
\hline anti-SEA & - & + & \pm & \pm & \pm & \pm & - \\
anti-SEB & - & - & +++ & ++ & - & ++ & - \\
anti SEC & + & - & - & +++ & - & \pm & - \\
anti-SEC & - & - & - & +++ & - & ++ & - \\
anti-SEC & - & - & - & +++ & - & \pm & ++ \\
anti-SED & - & \pm & \pm & \pm & + & +++ & - \\
anti-TSST-1 & - & - & - & - & - & - & ++ \\
anti-a-haemolysin & - & - & \pm & \pm & \pm & +++ & - \\
\hline
\end{tabular}

Reaction between antisera and polypeptide bands as assessed by immunoblotting following polyacrylamide gel electrophoresis

+++ is a strong reaction, - is a negative reaction.

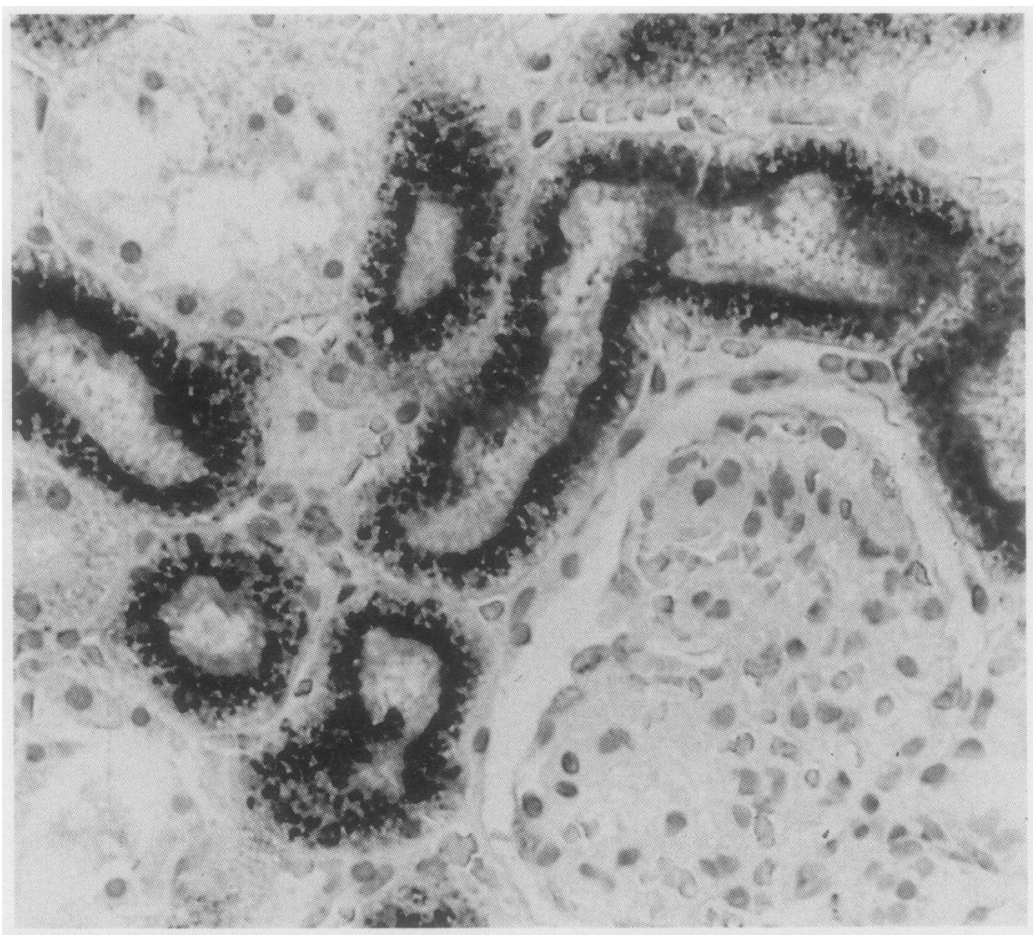

Figure 2 Immunoperoxidase technique using anti-SEB shows strong granular stainings in the proximal convoluted tubular cells of the rat kidney. This rat had been injected with $S E B$
The results of testing the products of a range of staphylococci against the staphylococcal antisera by immunoblotting are shown in the table. The antiserum to TSST- 1 reacted with TSST-1 alone. However, the other antisera showed rather more complex results. SEB and SEC stained strongly with their specific antisera, but SEA and SED stained less strongly with their specific antisera. Anti-SEC 1 stained a band of molecular weight 35000 and a number of antisera reacted with a band of molecular weight of 24000 . Anti-SEC 3 reacted with TSST-1 at a molecular weight of 22000.

Bacterial products of the staphylococcal strain Wood 46, which produces $a$-haemolysin, were examined by immunoblotting using anti$a$-haemolysin. Most of the bands showed weak positive staining indicating that the antiserum lacked specificity.

\section{IMMUNOHISTOLOGY IN RATS}

There was strong positive granular staining in the proximal convoluted tubular cells of the kidney in the rats injected with SEB, SEC, SED, and TSST-1 and then stained with specific antisera (fig 2). The control rats injected with saline were consistently negative. Furthermore, the toxins detected in the kidney by immunohistology did not show cross-reaction with other antisera. There was no detectable staining in the proximal convoluted tubular cells in rats given SEA and $a$-haemolysin and then examined with their specific antisera.

There was also staining of Kupffer cells in the liver (fig 3) and phagocytic cells in the spleen in rats given SEB, SEC, and TSST-1, but there was no staining in rats given SEA and $a$-haemolysin. The control rats given saline were negative and tests for cross-reactivity were also negative. There was no specific staining in the heart, thymus, skin, gut and lung apart from occasional positive phagocytic cells.

During the course of this work it was found that the best results were obtained if the rat kidney blocks were fixed for two hours at $37^{\circ} \mathrm{C}$ in formalin and then processed immediately. If the blocks were left overnight in formalin the staining intensity was considerably reduced. If the rat kidney was left overnight at $4^{\circ} \mathrm{C}$ and then processed following brief formalin fixation the staining intensity was not much diminished.

ELECTROPHORESIS OF RAT TISSUE HOMOGENATES Homogenates of rat kidney examined by SDSPAGE showed numerous superimposed bands and it was impossible to identify toxins by their position. Following immunoblotting and examination by specific antisera, however, it was possible to identify TSST-1 and SEB in the rats injected with these toxins (fig 4). Less consistently SEA, SEC, and SED have been identified in this way in rats given the appropriate toxin. The rats given saline were always negative. Homogenates of heart, liver, and spleen were also always negative. 


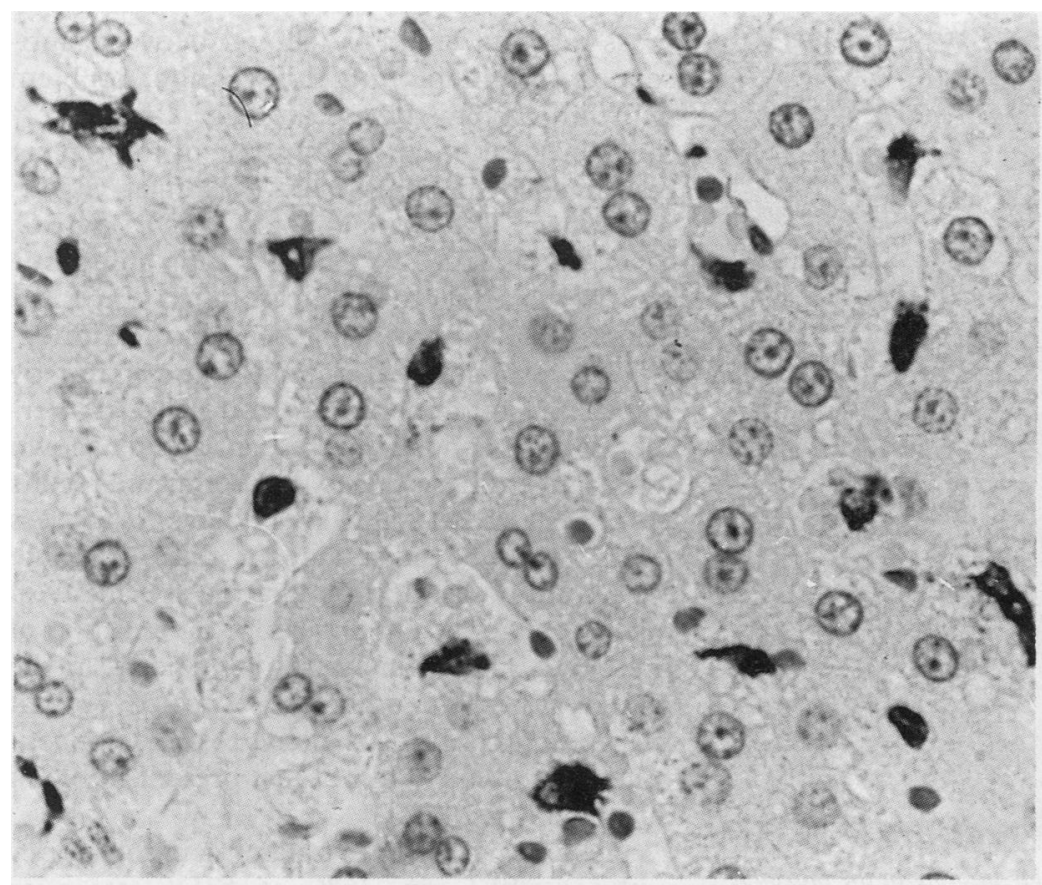

Figure 3 Immunoperoxidase technique using anti-SEC $C_{2}$ shows strong positive staining of Kupffer cells in the rat liver. This rat had been injected with SEC.

IMMUNOHISTOLOGY OF HUMAN ARCHIVAL MATERIAL Formalin fixed, paraffin wax embedded blocks of kidney and liver from 50 cases of SIDS and 50 other necropsy cases were retrieved from the files. Sections of each block were stained with the antisera to SEA, SEB. SEC ${ }_{2}$ and SED. Results with anti-TSST-1 have been reported previously. ${ }^{11}$ The antisera against $\mathrm{SEC}_{1}$ and $\mathrm{SEC}_{3}$ were not used as it had been found in the rats that they gave the same results as anti$\mathrm{SEC}_{2}$.

There was weak, but definite, granular staining in the proximal convoluted tubular cells of the kidney in 18 cases of SIDS and six controls with anti-SEC 2 (fig 5). This difference was significant $\left(\chi^{2}=6, \mathrm{p}<0.025\right)$. Staining with the other antisera in the kidney was consistently negative in both cases and controls. Kupffer cells did not stain with any antisera in either cases or controls.

In the comparison series there were 14 cases between 8 days and 3 years of age. Only one of these was positive, a child who died of staphylococcal septicaemia. The other five positive cases in the comparison group were in an older age range. The causes of death were myocardial infarction, status epilepticus, carbon monoxide poisoning, subarachnoid haemorrhage and pulmonary embolism.

There was no correlation between SEC positivity in the kidneys and staphylococcal isolation from the nasopharynx in SIDS. Sixteen of the 50 cases of SIDS had $S$ aureus isolated from the nasopharynx and only five (31\%) of these were positive for SEC. Of the 34 cases which were negative for $S$ aureus, 13 were positive for SEC (38\%).

The cases of SIDS had previously been stained for TSST-1 and nine were positive. There were four cases which were positive for TSST-1 and SEC.

\section{Discussion}

There is both theoretical and experimental evidence to support the idea that bacterial toxins could have a role in SIDS. The most plausible theory is a two stage model in which viral respiratory tract infection disturbs the normal microbial flora, leading to bacterial overgrowth and toxin absorption. ${ }^{1}$ The infants will only be susceptible to the lethal effects of the toxins in the short period between loss of maternal IgG and acquisition of specific

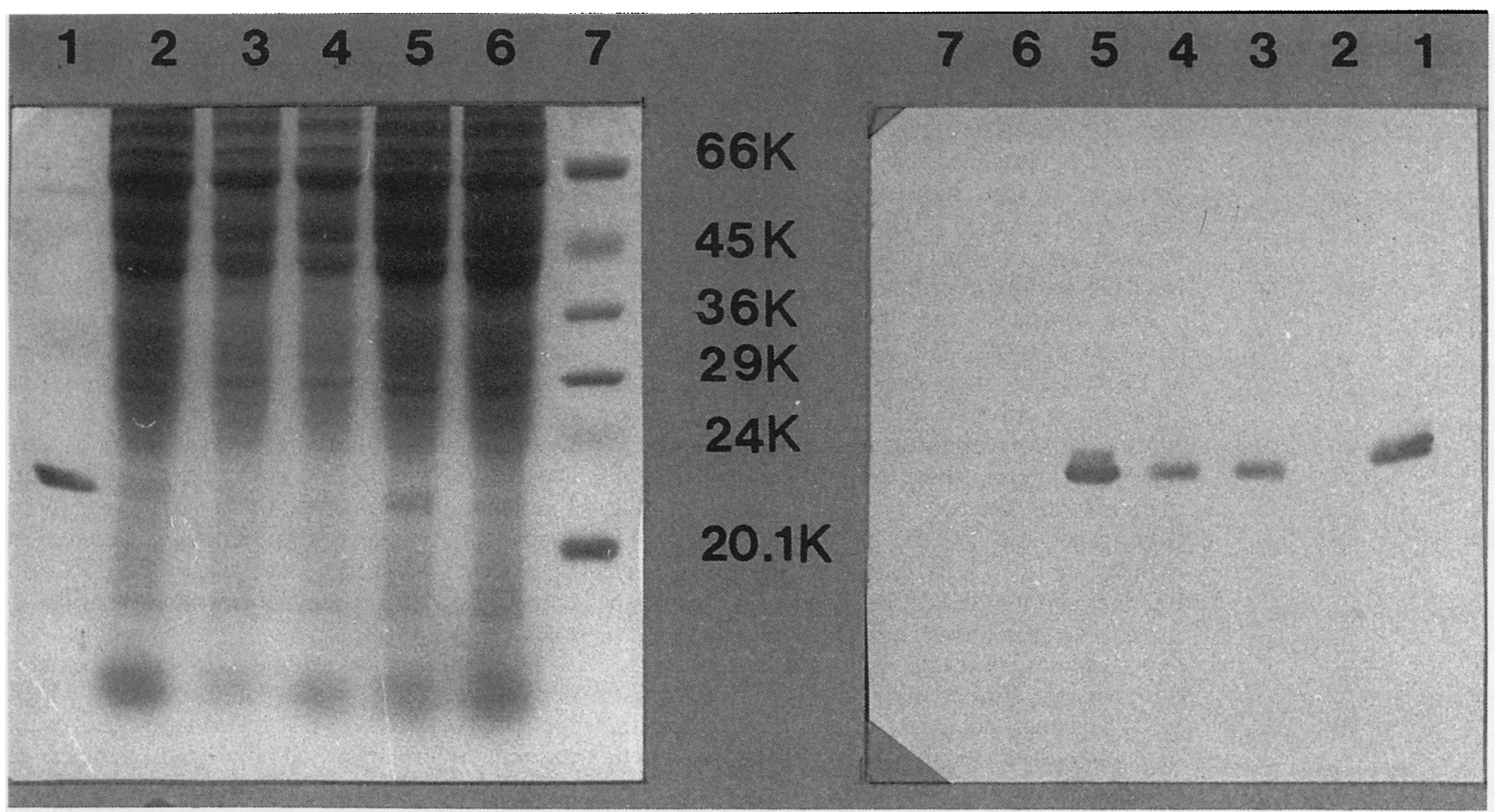

Figure 4 SDS-PAGE of rat kidney homogenates (lanes 2-6), standard markers (lane 7) and products from a staphylococcus known to produce TSST-1 (lane 1). The kidney homogenates in lanes 3, 4 and 5 were from rats injected with TSST-1. The mirror image immunoblot on the right is stained with anti-TSST-1. 


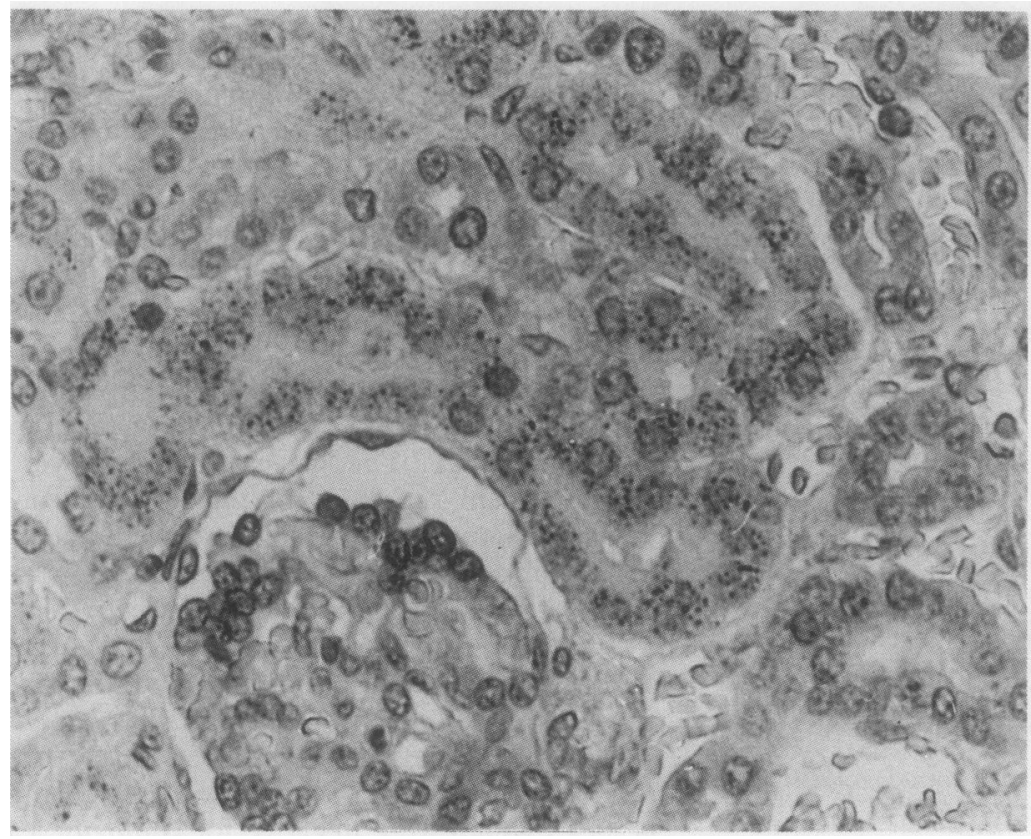

Figure 5 Kidney from case of SIDS showing positive granular staining in the proximal

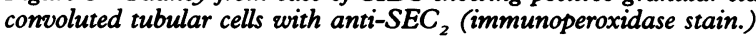

immunity. There is evidence of increased staphylococcal, streptococcal, and enterobacterial nasopharyngeal carriage in cases of SIDS compared with healthy infants of the same age. ${ }^{4}$ There is also evidence of increased gastrointestinal carriage of enterotoxigenic enterobacteria in SIDS. ${ }^{5} 7$ The model becomes more complicated when it is realised that bacteria can act in synergy to produce a lethal effect, ${ }^{810}$ and the action of their toxins can be potentiated by concomitant viral infection. ${ }^{9}$

To confirm this idea the presence of lethal toxins needs to be shown in body fluids and tissues of SIDS cases. This could prove difficult, however, for several reasons. The toxins might be present at low concentrations, they might be quickly absorbed by target cells, and other toxins might enter the bloodstream as an agonal event. The observation, therefore, that TSST- 1 is concentrated in the proximal tubular cells of the kidneys is of considerable theoretical interest. ${ }^{11}$ If other toxins are concentrated at this site investigation of their role in SIDS will be simplified.

The results of this work show that intravenously injected SEB, SEC, and SED, as well as TSST-1 are all concentrated in the proximal convoluted tubular cells of the kidney. This finding is not surprising as it has been known for many years that low molecular weight proteins are filtered by the glomerulus and then taken up by the proximal convoluted tubular cells. ${ }^{13}{ }_{14}$ In fact this has previously been shown for SEB. ${ }^{15}$ Because SEA is similar in size to the other enterotoxins it, too, is probably concentrated at this site. Failure to demonstrate this in the rats with immunohistology might be related to the fact that antiSEA only gave a weak reaction when examined by PAGE and immunoblotting. The anti$a$-haemolysin antiserum lacked specificity and no useful conclusion can be drawn about the fate of this molecule. Interestingly, although cross-reaction could be shown between antisera on immunoblotting this was not a problem with immunohistology in rats. Presumably formalin fixation impaired some of the weaker reactions so that they were no longer visible. There was also some uptake of SEB, SEC, SED, and TSST-1 by Kupffer cells and tissue phagocytes but the concentration in these organs was less noticeable than in the kidney.

The results of examining rat kidney by PAGE and immunoblotting were also of interest. TSST-1 and SEB were consistently shown in rats given these toxins, but so far the results with SEA, SEC, and SED have been less consistent. The advantage of this technique over immunohistology is that the specificity of of the reaction is confirmed by the position of the band. The toxins were only shown in the kidney, not in the other tissues tested, so that the finding confirms that the toxin is concentrated at this site and not just present in blood and interstitial fluid.

The finding of positive staining in the kidney with anti-SEC in $36 \%$ of SIDS cases as opposed to $12 \%$ of the comparison group is also interesting. The pattern of staining was similar to, but much less intense, than that found in rats given SEC intravenously. The comparison cases were not age matched, but there were 14 cases between 8 days old and 3 years of age and only one of these was positive, an infant who died of staphylococcal septicaemia. As in the previous work with TSST-1, however, there was no correlation between finding SEC in the kidney and isolating staphylococci from the nasopharynx. A possible explanation is that there is potential for extensive cross-reaction between toxins from different species so that a positive immunohistochemical stain with anti-SEC does not confirm specificity of the antigen. It is for this reason that methods using electrophoresis and immunoblotting are required.

In the immunohistological study of rat kidneys it was found that leaving rat tissue unfixed for variable periods after death did not impair staining, but prolonged fixation in formalin did. This has important practical implications. In SIDS the interval between death and discovery varies, with a further variable interval before the necropsy is performed. This need not impair immunostaining so long as the material is fixed in formalin for a short interval and then processed. The blocks used in this retrospective study, however, had been fixed in formalin for varying amounts of time, usually around 24 hours but sometimes much longer. Furthermore, although there is no reason to suspect that there is a systematic difference in formalin fixation time for cases of SIDS and comparison cases, we are not in a position to exclude the possibility.

The finding of antigens in the proximal convoluted tubular cells of the kidney which react with anti-TSST- $1^{11}$ and anti-SEC is of interest in relation to the pathogenesis of SIDS, particularly as this is the site at which many bacterial toxins are concentrated. There are too many problems, however, with a retrospective study of archival material for 
definite conclusions, but there is sufficient circumstantial evidence to warrant an intensive study of the kidney in SIDS. This should be done prospectively, with optimal fixation, and using a wide range of antisera to bacterial toxins. In parallel, kidney homogenates should be examined by electrophoresis and immunoblotting to confirm specificity of staining. Furthermore, because antisera to all potentially important bacterial toxins are not available, it will be important to develop bioassay systems for lethal toxins using kidney homogenates.

This work was funded by a research grant from North Western Regional Research Committee.

1 Morris JA, Haran D, Smith A. Hypothesis: Common bacterial toxins are a possible cause of the sudden infant death syndrome. Medical Hypotheses 1987;22:211-22.

2 Morris JA. Increased risk of sudden infant death syndrome in older infants at weekends. Br Med F 1986;293:566.

3 Morris JA. Sudden infant death syndrome. $\mathrm{Br}$ Med $\mathrm{f}$ 1989;298:958.

4 Telford DR, Morris JA, Hughes $P$, et al. The nasopharyngeal Telford DR, Morris JA, Hughes $P$, et al. The nasopharyngeal
bacterial flora in sudden infant death syndrome. $\mathcal{f}$ Infect $1989 ; 18: 125-30$

5 Bettelheim KA, Goldwater PN, Dwyer BW, Bourne AJ, Smith DL. Toxigenic Escherichia coli associated with sudden infant death syndrome. Scand $\mathcal{F}$ Infect Dis 1990;22:467-76.
6 Arnon SS, Midura TF, Damus K, Wood RM, Chin J. Intestinal infection and toxin production by Clostridium botulinum as one cause of sudden infant death syndrome. Lancet 1978 ;i:1273-7.

7 Sonnabend OAR, Sonnabend WFF, Krech U, Molz G, Sigrist T. Continuous microbiological and pathological study of 70 sudden and unexpected infant deaths: Toxigenic intestinal Clostridium botulinum infection in 9 cases of sudden infant death syndrome. Lancet $1985 ; \mathrm{i}: 237-41$

8 Lee S, Barson AJ, Drucker DB, Morris JA, Telford DR. Lethal challenge of gnotobiotic weanling rats with bacterial isolates from cases of sudden infant death syndrome. f Clin Pathol 1987;40:1393-6.

9 Jakeman KJ, Rushton DI, Smith H, Sweet C. Exacerbation of bacterial toxicity to infant ferrets by influenza virus: Possible role in sudden infant death syndrome. $\mathcal{F}$ Infect $D$ is Possible role in sudd

10 De Azavedo JCS, Lucken RN, Arbuthnott JP. Effect of toxic shock syndrome Toxin-1 on chick embryos. Infect Immun 1985;47:710-12.

11 Newbould MJ, Malam J, McIllmurray JM, Morris JA, Telford DR, Barson AJ. Immunohistological localisation of staphylococcal toxic shock syndrome toxin (TSST-1) antigen in sudden infant death syndrome. $f$ Clin Pathol 1989;42:935-9.

12 Laemmli UK. Cleavage of structural proteins during the assembly of the head of bacteriophage T4. Nature 1970;227:680-5.

13 Straus W. Cytochemical observations on the relationship between lysosomes and phagosomes in kidney and liver by combined staining for acid phosphatase and intravenously injected horseradish peroxidase. I Cell Biol venously injected

14 Norman SJ. Renal fate of the staphylococcal enterotoxin B. Lab Invest 1971;25:126-32.

15 Norman SJ, Jaeger RF, Johnsey RT. Pathology of experimental enterotoxaemia: the in vivo localisation of staphylococcal enterotoxin B. Lab Invest 1969;20:17-25. 